\title{
Whole genome sequencing and assembly of a Caenorhabditis elegans genome with complex genomic rearrangements using the MinION sequencing device
}

Authors: Tyson $\mathrm{JR}^{1^{*}}$, O’Neil $\mathrm{NJ}^{2 *}$, Jain $\mathrm{M}^{3^{*}}$, Olsen $\mathrm{HE}^{3}$, Hieter $\mathrm{P}^{4}$, Snutch $\mathrm{TP}^{1}$

1. Michael Smith Laboratories and Djavad Mowafaghian Centre for Brain Health, University of British Columbia, Vancouver, Canada.

2. Michael Smith Laboratories, University of British Columbia, Vancouver, BC, Canada V6T $1 Z 4$

3. UC Santa Cruz Genomics Institute and Department of Biomolecular Engineering, University of California, Santa Cruz, California, USA.

4. Michael Smith Laboratories, University of British Columbia, Vancouver, BC, Canada V6T 1Z4; Department of Medical Genetics, University of British Columbia, Vancouver, BC, Canada V6T $1 Z 3$

\section{ABSTRACT}

Advances in $3^{\text {rd }}$ generation sequencing have opened new possibilities for 'benchtop' whole genome sequencing. The MinION is a portable device that uses nanopore technology and can sequence long DNA molecules. MinION long reads are well suited for sequencing and de novo assembly of complex genomes with large repetitive elements. Long reads also facilitate the identification of complex genomic rearrangements such as those observed in tumor genomes. To assess the feasibility of the de novo assembly of large complex genomes using both MinION and Illumina platforms, we sequenced the genome of a Caenorhabditis elegans strain that contains a complex acetaldehyde-induced rearrangement and a biolistic bombardment-mediated insertion of a GFP containing plasmid. Using $~ 5.8$ gigabases of MinION sequence data, we were able to assemble a $C$. elegans genome containing 145 contigs $(\mathrm{N} 50$ contig length $=1.22 \mathrm{Mb})$ that covered $>99 \%$ of the $100,286,401$ bp reference genome. In contrast, using $\sim 8.04$ gigabases of Illumina sequence data, we were able to assemble a C. elegans genome in 38,645 contigs (N50 contig length $=\sim 26 \mathrm{~kb}$ ) containing $117 \mathrm{Mb}$. From the MinION genome assembly we identified the complex structures of both the acetaldehyde-induced mutation and the biolistic-mediated insertion. To date, this is the largest genome to be assembled exclusively from MinION data and 
is the first demonstration that the long reads of MinION sequencing can be used for whole genome assembly of large $(100 \mathrm{Mb})$ genomes and the elucidation of complex genomic rearrangements.

\section{Introduction}

Advances in Next Generation Sequencing (NGS) have ushered in a new era of whole genome analysis. The short sequencing reads generated by sequencing-by-synthesis NGS are well suited for resequencing complex genomes for which a reference sequence has been established. However, short reads are poorly suited for de novo assembly of complex genomes in part due to repeat regions that generate highly discontinuous assemblies and require unique flanking sequences to position repeat elements. In many instances if a sequencing read does not completely span a repeat, either due to repeat size or physical properties of the repetitive DNA, it can not be unambiguously assembled into a contig. This can pose a problem for the de novo assembly of metazoan genomes where repetitive elements are common. For example, approximately $12 \%$ of the $100 \mathrm{Mb}$ C. elegans genome is derived from transposable elements (Bessereau JL. 2006). As such, NGS is not optimal for sequencing and assembly of large novel genomes or genomes with numerous complex rearrangements such as those observed in tumour cells.

Third Generation Sequencing (TGS) technologies have increased read lengths 100 to 1000 -fold compared to NGS platforms and therefore can span much larger repeat regions than NGS. The first major TGS platform was the Pacific Biosciences Single Molecule Real Time (SMRT) PacBio sequencing system. PacBio sequencing averages $10 \mathrm{~kb}$ read lengths and with consensus sequencing the error rate approaches that of NGS. In 2014, Oxford Nanopore Technologies (ONT) introduced the MinION nanopore sequencer. The MinION directly connects to a laptop or desktop PC via a USB3 port, is $\sim 90$ grams, and costs are for reagents only. The MinION works on the principle of nanopore strand sequencing (Deamer et al. 2016). Briefly, a DNA strand is enzymatically unzipped and ratcheted through a membrane-inserted protein nanopore as a voltage potential is applied. As the DNA single strand translocates, the resulting changes in ionic current are measured and sequence calls produced corresponding to the identity of the 
nucleotides via computationally inferred model fitting. When only one strand (template) from a duplex DNA molecule is read by the nanopore, the resulting sequence call is termed a 1D read (Jain et al 2016). With certain library preparations an optional hairpin strand bridge provides the opportunity to sequence both strands of a dsDNA (template and complement) generating a 2D read, which results in a higher accuracy sequence call. MinION sequence read lengths range from several hundred bases to hundreds of thousands of bases, and are essentially limited by the DNA preparation type and delivery to the pore. Early versions of the MinION sequencing chemistry and base calling produced sequences with relatively high error rates $(22-35 \%)$ when compared to both NGS $(<2 \%)$ and PacBio TGS (10-15\%). The high error rate combined with relatively low sequencing yields per flow cell $(<1 \mathrm{~Gb} /$ flow cell) led some researchers to underestimate the potential for this technology. Recent advances in the MinION chemistry and base calling have greatly improved accuracy (5-10\% error rate) and yield (2-5 Gb/flowcell) (J. Tyson unpublished results). To date, most studies have used the MinION to sequence small genomes or to partially survey larger genomes to assess chromosomal structure and copy number variations (Loman et al. 2015, Goodwin et al. 2015, Norris et al. 2016, Wei \& Williams 2016,). The long read lengths of this technology, combined with recent improvements in performance make the MinION a viable option for whole genome sequencing of complex metazoan genomes.

The Caenorhabditis elegans genome was the first metazoan genome to be completely sequenced (C. elegans Sequencing Consortium 1998) and is an excellent model genome for assessing new whole genome sequencing technologies. The C. elegans genome is complex with many different types of local and dispersed repeat sequences in both intronic and intergenic regions and only $27 \%$ of the genome residing in exons. Local repeats range from short repetitive sequences such as homopolymeric $\mathrm{G}$ tracts (Zhao et al. 2007) to large tandem repeats spanning tens of kilobases. The most common dispersed repeats are derived from transposable elements and constitute approximately $12 \%$ of the genome (Bessereau 2006). C. elegans transposons range in size from 1-3 $\mathrm{kb}$ and can confound genomic assemblies as transposon sequences are larger than NGS and Sanger sequencing reads resulting in ambiguous mapping positions. The assembly of the $C$. elegans reference genome was made possible by the construction of a high quality physical map of cosmids and yeast artificial chromosomes (Coulson et al. 1988; Coulson et al. 1995; Coulson 
et al. 1991), which were individually shotgun sequenced and manually finished to bridge gaps and ambiguous regions to generate a complete high quality reference genome $(\mathrm{C}$. elegans Sequencing Consortium 1998). The high-quality reference genome has facilitated studies using NGS re-sequencing to aid identifying new mutants and analysing mutational profiles (Thompson et al. 2013; Meier et al. 2014). Nevertheless, the potential for assembly discrepancies using NGS in sequence determination remain and NGS is not optimal for de novo genome assembly or understanding large structural alterations such as those associated with cancer and complex genetic disorders.

Here, we report the sequencing and de novo assembly of a $100 \mathrm{Mb}$ C. elegans genome exclusively from MinION reads. For comparison, we used both MinION and Illumina platforms to sequence the genome of a $C$. elegans strain containing both a complex genome rearrangement generated by acetaldehyde mutagenesis and a ballistic-mediated insertional event of a GFPcontaining plasmid (him-9(e1487);ruIs32). The de novo assembly of MinION data generated a remarkably complete genome in 145 contigs that cover $>99 \%$ of the reference genome with an $\mathrm{N} 50$ contig length of $1.22 \mathrm{Mb}$. Furthermore, the sequence assembly elucidated the complex structure of the rearrangement and insertion events that were not readily apparent in the Illumina data. The strategy of comparison to Illumina data was however useful to improve the sequence accuracy of the MinION assembly. Together, this hybrid sequencing approach generated a physical map with high sequence accuracy and further represents the largest genome assembled exclusively from MinION data.

\section{Materials and Methods}

\section{Nematode culture and DNA extraction}

Nematodes were cultured as previously described (Brenner 1974). The him-9(e1487) II; unc119(ed3) ruIs32[pie-1p::GFP::H2B + unc-119(+)] III strain was constructed by mating CB1487 him-9(e1487) males to AZ212 unc-119(ed3) ruIs32 III hermaphrodites. F1 heterozygotes were selfed and him-9; ruIs32 homozygotes isolated. Worms were grown to starvation for sequencing on NGM plates and harvested by washing with M9 buffer and pelleted in 15-ml centrifuge tubes. Buffer was removed by two washes with sterile distilled water, centrifugation and aspiration. 
The worm pellet was resuspended in $300 \mu \mathrm{l}$ of lysis buffer $(200 \mathrm{mM} \mathrm{NaCl}, 100 \mathrm{mM}$ Tris- $\mathrm{HCl} \mathrm{pH}$ 8.5, $50 \mathrm{mM}$ EDTA $\mathrm{pH} 8.0,0.5 \% \mathrm{SDS}, 0.1 \mathrm{mg} / \mathrm{ml}$ proteinase $\mathrm{K}$ ) and frozen at $-80{ }^{\circ} \mathrm{C}$. Frozen pellets were incubated at $60{ }^{\circ} \mathrm{C}$ for $1-3$ hours followed by $95^{\circ} \mathrm{C}$ for 20 minutes. RNase A $(0.1$ $\mathrm{mg} / \mathrm{ml}$ ) was added and lysed worms were incubated at $37^{\circ} \mathrm{C}$ for 1 hour. DNA was prepared by standard phenol/chloroform extraction and DNA was resuspended in $10 \mathrm{mM}$ Tris $\mathrm{pH}$ 8.0.

\section{Library Preparation and MinION Sequencing}

The Chip84-Chip90 MinION flowcells were run using libraries prepared with the SQK-NSK007 Nanopore Sequencing Kit R9 version. Chip94 \& Chip95 libraries were prepared using the SQKRAD001 Rapid Sequencing Kit I R9 version. The standard protocols from Oxford Nanopore Technologies were used with the following modifications. For SQK-NSK007 libraries, purification of DNA after the FFPE treatment step was done using 0.4x AMPureXP beads. Prior to adapter ligation each elution step off the AMPureXP beads was performed using $10 \mathrm{mM}$ Tris $\mathrm{pH} 8.0$, instead of water, at $37^{\circ} \mathrm{C}$ for 5 mins. The starting amounts of gDNA ranged between 0.8 $\mu \mathrm{g}$ and $2.0 \mu \mathrm{g}$ (see Table S1 for details). Priming of individual flowcells with running buffer (2x $500 \mu \mathrm{l})$ and sequencing library top ups $(150 \mu \mathrm{l})$ were performed at times detailed in Table 1. Flowcells were run for $\sim 48 \mathrm{hrs}$ using custom device tuning scripts. The tuning scripts provide event yield monitoring aimed at maintaining data throughput through initiation of a maximal pore channel assignment/usage strategy and optimal bias-voltage selection via methods outlined below.

\section{Modified MinION running scripts}

After initial start of a MinION sequencer run using the standard ONT sequencing scripts, MinION sequencing control was shifted to a custom MinKNOW MinION script to enhance pore utilisation and increase data yields. This custom script adjusted a number of run/flowcell metrics and parameters including-

1) Initiation of a bias-voltage re-selection and active pore re-population into active channels when the hourly event yields falls below a threshold. This threshold was set at $67 \%$ of the first hour of each particular sequencing segment, and generally ran for 2-5 hours per segment. 
2) Identifying the bias-voltages that provide the greatest number of active pores by scanning a voltage range (20-30 $\mathrm{mV}$ in increments of -10 mV) and using this for active pore channel reassignment. A newly selected bias-voltage acts as the starting point for subsequent scans, and provides an active pore "tracking" ability as the required bias-voltage magnitude increases during a run with the electrochemical gradient decay of individual wells. This re-assignment also provides access to the full 2048 possible wells repeatedly throughout a run.

3) Selecting a lower magnitude bias-voltage wherein the active pore numbers are within $10 \%$ of the peak voltage. Keeping greater pore numbers active using these approaches results in wells/pores running for different periods of time and the bias-voltage requirement to drive the same current through a pore increase and diverge with use. This is because the electrochemical gradient of active wells/pores decays at a greater rate than that of inactive wells/pores. By using off peak, lower magnitude bias-voltage selection, a measure of pore population containment or "shepherding" is provided by moving lower magnitude voltage requiring pores into the rest of the population.

For further details on these device running script modifications see, https://community.nanoporetech.com/posts/r9-tuning-scripts-for-mink, and links contained within.

\section{Base-calling MinION Sequencing Reads}

All reads generated from the MinION sequencing device were base-called using the cloud-based Metrichor service provided by Oxford Nanopore. Details of specific versions can be found in Table S1 for the different runs. DNA sequences were extracted from individually called reads using simple python scripts and combined in a single fasta file format of a particular strand sequence type. Runs using the SQK-NSK007 (2D) kit generated template and a fraction of complement and 2D sequence from individual reads. SQK-RAD001 (1D) Library runs generated template (1D) reads. Filtering based on a quality metric by Metrichor divided the reads additionally into 'pass' and 'fail' categories. Some or all these sequence containing files for each run were then used for genome assembly as indicated. 


\section{Genome assembly and evaluation}

\section{SPAdes Illumina assembly}

For Illumina data, low quality bases were trimmed from both ends using seqtk (lh. lh3/seqtk. GitHub Available at: https:/github.com/lh3/seqtk. (Accessed: 26th November 2016)). The threshold used for trimming was a quality score of 30. We performed an Illumina only assembly using SPAdes (Nurk et al. 2013) in its default settings with kmer sizes 21, 51, and 71. The number of threads used was 32 with a memory of 156 GB.

\section{Canu nanopore assembly}

We pooled all the nanopore data (pass and fail; 1D and 2D) and filtered out reads below a size of $1 \mathrm{~kb}$ to avoid overlap detection issues. The remainder of reads ( $\sim 6.03$ gigabases) were assembled using Canu (Koren et al. 2016) in its default settings.

\section{Assembly correction}

The Canu assemblies were corrected using Pilon (Walker et al. 2014) using recommended settings to polish for variants and homopolymers. We did not perform quality filtering on the Illumina data and all of the $\sim 8.04$ gigabases of short read sequence was used.

\section{Nanopore data and assembly evaluation}

Nanopore reads and assemblies were evaluated by alignment against the reference worm genome using marginAlign version 0.1 (with BWA-MEM (Li 2013)) (Jain et al. 2015). Alignment and error statistics were computed using marginStats version 0.1 (Jain et al. 2015). To better estimate the errors for nanopore data, we improved the alignments using marginAlign EM (Jain et al. 2015). We also performed secondary evaluations using LASTZ alignments to align draft assemblies against the reference.

We evaluated the assembly quality by using QUAST (Gurevich et al. 2013) with recommended settings and the reference worm genome. We compared changes in indels and mismatches for the Canu assemblies before and after Pilon correction. We evaluated the assemblies based on: 1) the total number of aligned bases in the assembly; 2) the number of mismatches; and 3) the total number of bases contained in indels. 


\section{Results}

\section{Sequencing a $C$. elegans strain with complex rearrangements}

To assess the feasibility of using MinION sequencing to both generate de novo whole genome assemblies of large genomes and delineate complex rearrangements, we constructed for sequencing a C. elegans strain, him-9(e1487) II; unc-119(ed3) ruIs32[pie-1p::GFP::H2B + unc$119(+)$ ] III, that contained two homozygous complex rearrangements. The him-9(e1487) mutation was induced by acetaldehyde mutagenesis (Hodgkin et al. 1979) and is a complex duplication and insertion event that disrupts the predicted C. elegans XPF orthologue $x p f-1$ (Youds et al. 2006). Previous analysis of him-9(e1487) determined that the insertion contained duplicated sequence from the mab-3 gene (N.J. O'Neil, unpublished data). However, the complex nature of the mutation stymied attempts to determine the exact structure of the rearrangement. The ruIs32 insertion is a low-copy number insertion that was generated by biolistic transformation of the plasmid pAZ132 [Ppie-1::GFP::H2B::pie-1] (derived from pJH4.52) and a plasmid containing unc-119 [unc-119(+)] into an unc-119(ed3) mutant (Wormbase). Genomic DNA was prepared from the him-9(e1487) II; ruIs32 III strain and sequenced by both a MinION sequencer using R9 chemistry and Illumina sequencing using 300 base paired-end reads.

Sequencing runs from six MinION flow cells using the R9.0 (4x) and R9.3 (2x) pore flowcell types were produced on the MinION device controlled by custom tuning scripts (see Methods). This resulted in $1.1 \mathrm{M}$ individual reads up to 123,159 bases in length (mean $=4,801$ ) and containing $5.33 \mathrm{~Gb}$ of $1 \mathrm{D}$ bases. An additional $1 \mathrm{~Gb}$ of $2 \mathrm{D}$ sequence was generated from the paired template and complement 1D reads produced from the R9.0 flow cells using the SQKNSK007 2D chemistry and Metrichor basecaller. Details of the individual MinION sequencing runs and the MinION sequencing chemistries used can be found in (Figure 1) and (Table 1). Significant improvements in the 1D sequence quality of individual reads was observed when comparing R9.3 to R9.0 (Figure S1). This evolution on a path to the present day R9.4 pore provided significant advances in the simpler production of higher quality 1D reads approaching the percentage read identity of the previous generation 2D reads. Nanopore read quality was measured by alignment to the C. elegans reference genome. The median identity for pass 1D 
bioRxiv preprint doi: https://doi.org/10.1101/099143; this version posted January 30, 2017. The copyright holder for this preprint (which was not certified by peer review) is the author/funder, who has granted bioRxiv a license to display the preprint in perpetuity. It is made available under aCC-BY-NC-ND 4.0 International license.

\section{D Library Prep - R9.0}

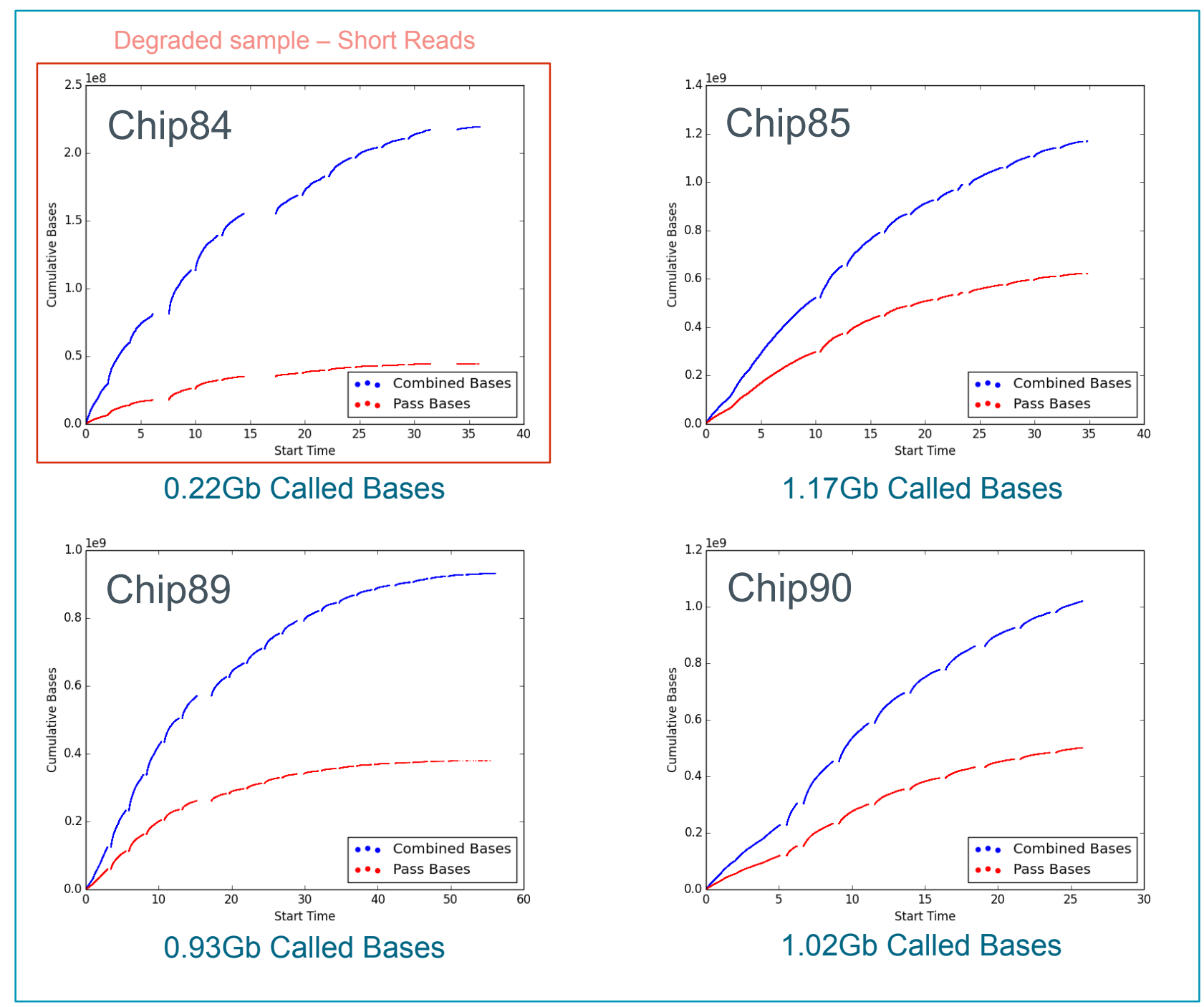

1D Rapid Prep - R9.3
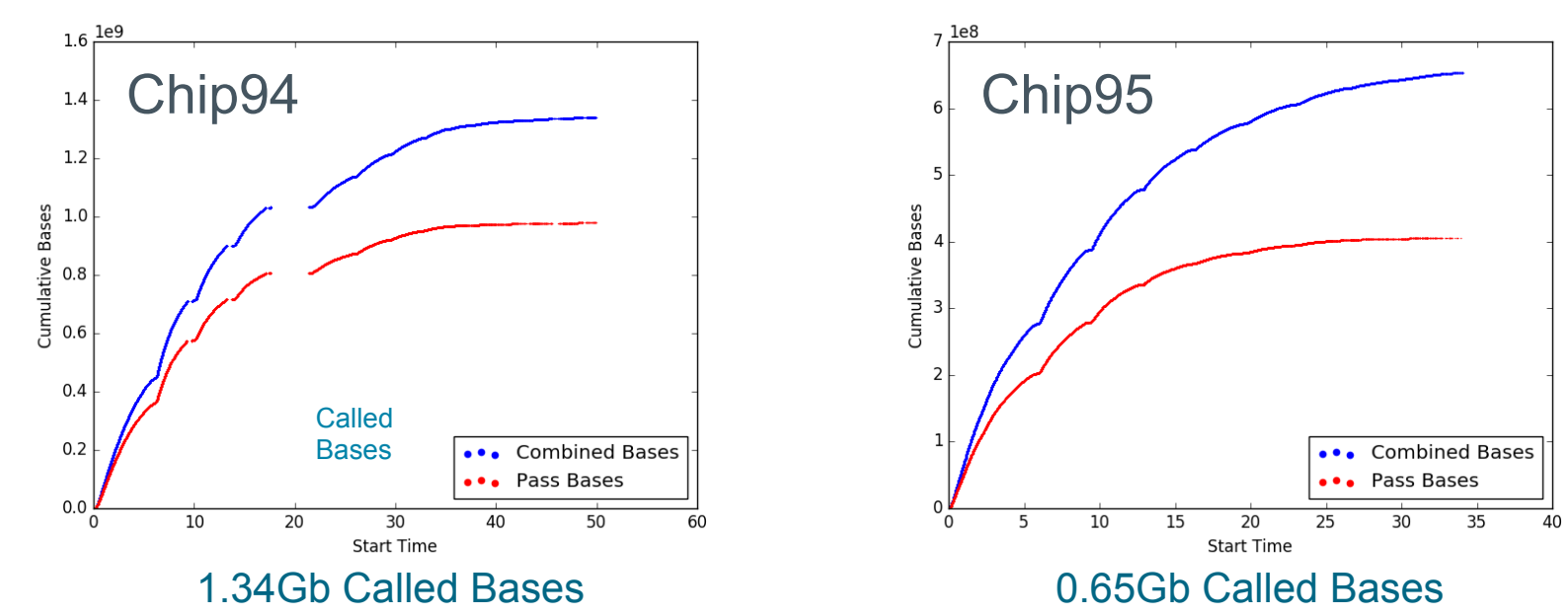

Figure 1. MinION Sequencing Run Summaries. Called base yield accumulations from MinION flowcell sequencing runs. 


\begin{tabular}{|c|c|c|c|c|c|c|}
\hline & Chip84 & Chip85 & Chip89 & Chip90 & Chip94 & Chip95 \\
\hline Library Kit & SQK-NSK007 & SQK-NSK007 & SQK-NSK007 & SQK-NSK007 & SQK-RAD001 & SQK-RAD001 \\
\hline Flowcell ID & FAD11292 & FAD11271 & FAD11308 & FAB23558 & FAD24831 & FAD24186 \\
\hline Pore Type & R9.0 & R9.0 & R9.0 & R9.0 & R9.3 & R9.3 \\
\hline Genomic DNA Start Material & 749ng & 1650ng & 1978ng & 1978ng & 864 ng into $20 u l$ & 1152 ng into $20 u l$ \\
\hline Volume Loaded & $12 \mathrm{ul} 0 \mathrm{hrs}, 12 \mathrm{ul} 0.5 \mathrm{hrs}$ & $12 \mathrm{ul} \mathrm{Ohrs,}$ & $12 \mathrm{ul} \mathrm{Ohrs}$ & $6 u l$ Ohrs, 6ul 24hrs & $11 \mathrm{ul} 0 \mathrm{hrs}, 11 \mathrm{ul} 24 \mathrm{hrs}$ & $11 \mathrm{ul} 0 \mathrm{hrs}, 11 \mathrm{ul} 24 \mathrm{hrs}$ \\
\hline Preseq Mix Conc. & $7.14 \mathrm{ng} / \mathrm{ul}$ & $5.34 \mathrm{ng} / \mathrm{ul}$ & $8.56 \mathrm{ng} / \mathrm{ul}$ & $8.56 \mathrm{ng} / \mathrm{ul}$ & na & na \\
\hline PlatQC Pores & $1155 / / 486,397,217,55$ & $1036 / / 467,357,170,42$ & $1060 / / 428,340,222,70$ & $1404 / / 489,444,330,141$ & $1277 / / 494,423,271,89$ & $1361 / / 504,448,312,97$ \\
\hline Total Reads & 198,913 & 184,600 & 142,374 & 155,508 & 268,004 & 161,378 \\
\hline Pass Reads & 36,949 & 68,204 & 34,816 & 54,892 & 180,938 & 89,376 \\
\hline Av Template Read Length & 831 & 4,052 & 4,486 & 4,430 & 5,001 & 4,049 \\
\hline Av Complement Read Length & 272 & 2,281 & 2,059 & 2,125 & na & na \\
\hline Av 2D Read Length & 171 & 2,207 & 1,826 & 1,972 & na & na \\
\hline Max Pass Template Read size & 9,460 & 29,221 & 44,484 & 32,910 & 108,915 & 123,159 \\
\hline Max Pass Complement Read size & 9,130 & 28,814 & 34,881 & 31,458 & na & na \\
\hline Max Pass 2D Read size & 9,740 & 29,681 & 44,405 & 33,553 & na & na \\
\hline Top 25\% Templates > & 904 & 6,106 & 6,858 & 6,622 & 6,390 & 4,717 \\
\hline Top 25\% Complements > & 305 & 4,233 & 2,721 & 2,896 & na & na \\
\hline Top 25\% 2D > & 273 & 4,454 & 1,987 & 2,533 & na & na \\
\hline Total Called bases & $219,411,804$ & $1,169,061,304$ & $931,927,653$ & $1,019,278,312$ & $1,340,369,063$ & $653,447,905$ \\
\hline 2D Pass Bases & $22,208,070$ & $323,598,976$ & $195,759,566$ & $258,533,514$ & na & na \\
\hline 2D fail Bases & $11,877,828$ & $83,731,694$ & $64,225,734$ & $48,089,637$ & na & na \\
\hline 2D Bases & $34,085,898$ & $407,330,670$ & $259,985,300$ & $306,623,151$ & na & na \\
\hline Base Calling & 2D Chimera 1.22 .10 & 2D Chimera 1.22 .10 & 2D Chimera 1.22 .10 & 2D Chimera 1.22 .10 & 1D Chimera 1.22.10 & 1D Chimera 1.22 .10 \\
\hline MinKNOW version & 0.51.3.55 & 0.51.3.55 & 0.51.3.55 & 0.51.3.55 & 1.0 .5 & 1.0 .5 \\
\hline Comment & Small Frags degraded Library & & & SpotON Flowcell & & \\
\hline
\end{tabular}

Table 1. MinION Sequencing Data Summary. Individual library preparation and run statistics for MinION sequencing of C.elegans him-9 mutant. 
bioRxiv preprint doi: https://doi.org/10.1101/099143; this version posted January 30, 2017. The copyright holder for this preprint (which was not certified by peer review) is the author/funder, who has granted bioRxiv a license to display the preprint in perpetuity. It is made available under aCC-BY-NC-ND 4.0 International license.
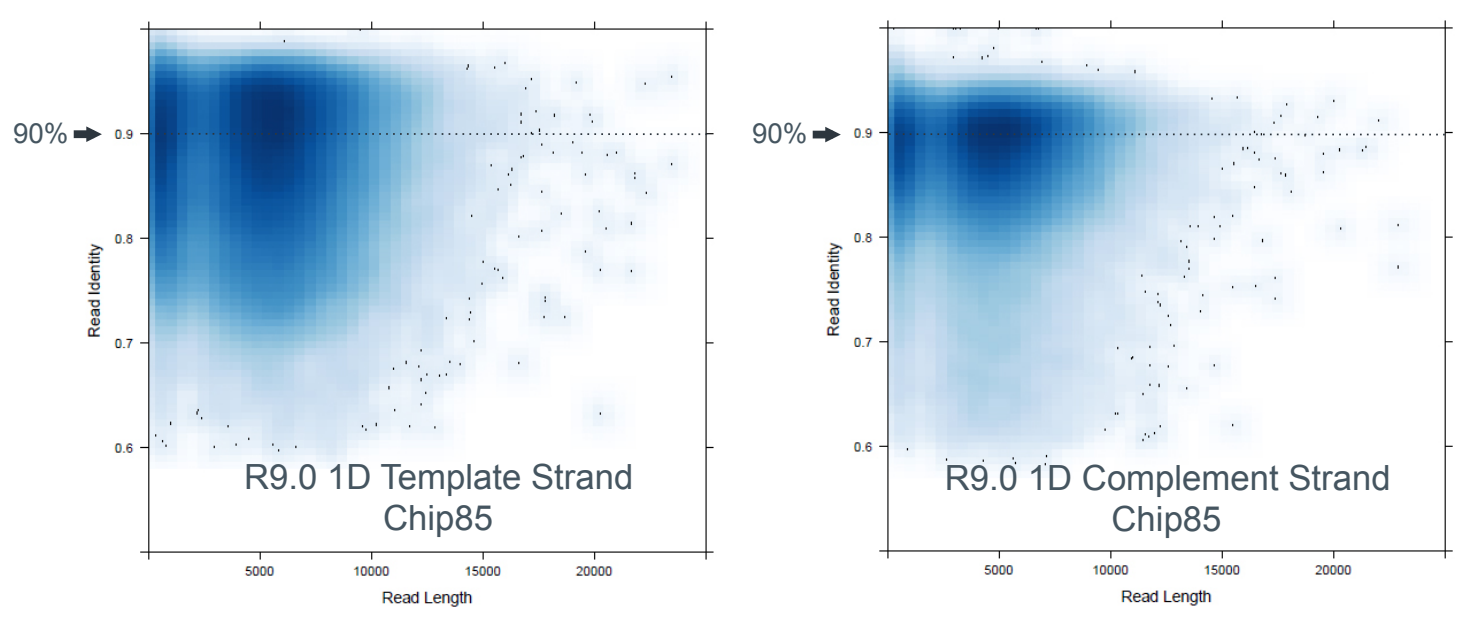

$90 \%$

$90 \%$
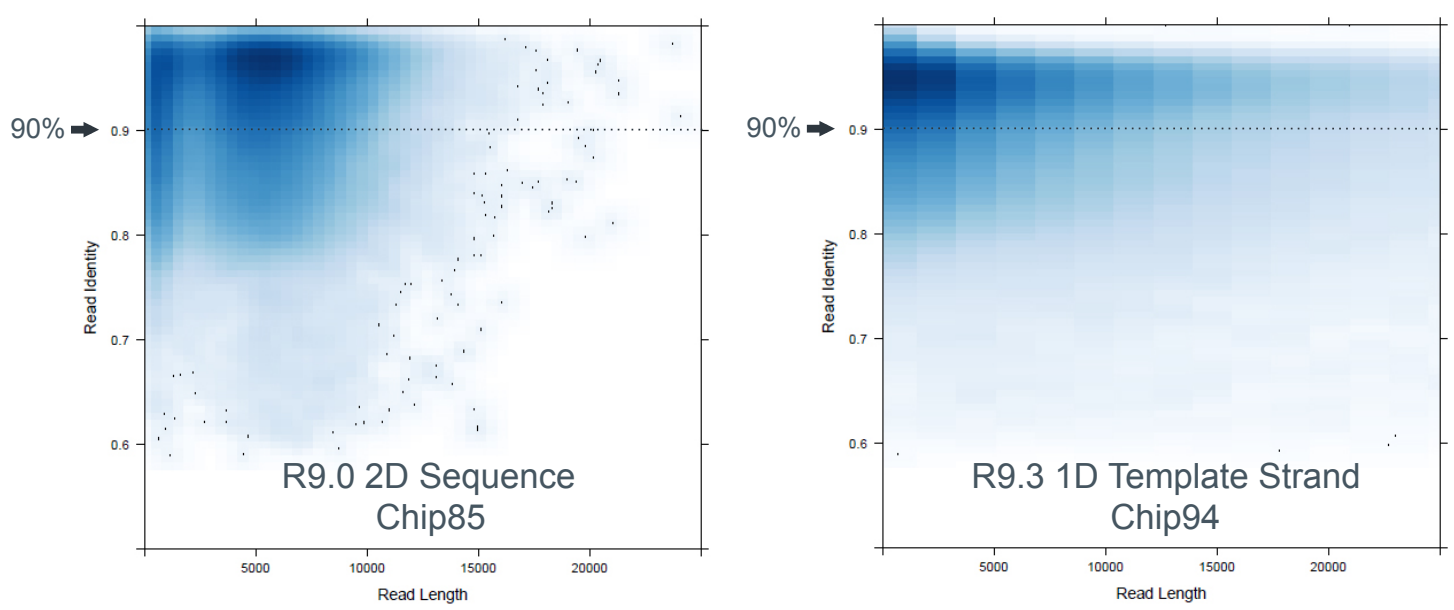

Figure S1. MinION Individual Read Accuracies vs Read Length. Example plots for both R9.0 2D and R9.3 1D chemistries. Individual pass read accuracies from aligned regions calculated using bwa alignment to the reference sequence and plotted against individual read length. 
reads from the 1D runs that used SQK-RAD001 Rapid Sequencing Kit I R9 version was $\sim 93 \%$. The median identities for pass 2D reads from the 2D runs that used SQK-NSK007 Nanopore Sequencing Kit R9 version (Chips 84-90) ranged between 90-95\%. Illumina sequencing produced 14,041,499 paired end reads totaling $\sim 8.04 \mathrm{~Gb}$ of sequence (Table 2). Alignment of the MinION sequence reads to the $C$. elegans reference genome demonstrated that most of the genome was well covered ( $\sim 50 \mathrm{X}$ coverage) and identified an apparent $\sim 2 \mathrm{Mb}$ duplication on chromosome III (Figure 2).

\section{de novo assembly of the $C$. elegans genome from MinION and Illumina sequence reads}

$1 \mathrm{D}$ and $2 \mathrm{D}$ sequence reads were filtered by size to exclude reads $<1 \mathrm{~kb}$ and Canu (Korin et al 2016) a genome assembler designed for high-noise single-molecule sequencing was used to assemble contigs. A Canu assembly was performed using all "pass" and "fail" reads from all prepared library types to generate an assembly containing 294 contigs ranging in length from 3,695 to $4,573,586$ bases with an average contig size of $351 \mathrm{~kb}$. The Canu contigs were polished using Pilon and the Illumina sequence data (Table 3). 145 of the contigs containing 101,982,548 bases had significant homology to C. elegans (Table 4). Most of the non-C. elegans contigs were homologous to bacterial genomes consistent with the bacteria present on the NGM petri plates used to grow the nematodes. The mean length for C. elegans matching contigs was 703,328 bases compared to a mean contig length of 28,266 bases for the 149 non-C. elegans contigs, likely due to lower coverage. The 145 C. elegans contigs were aligned to the WBcel235 release of the C. elegans reference genome using LASTZ aligner (Harris, R.S. 2007). The alignment of contigs to the six chromosomes and the C. elegans mitochondrial genome covered $>99 \%$ of the reference genome with $>97 \%$ identical sites (Table 5; Figure 3). There were no large gaps in coverage of the reference genome. Three contigs, aligned to two different chromosomes resulting in apparent discontinuities. These hybrid contigs were most likely misassembled contigs as no other evidence suggests that these regions have been translocated.

\section{MinION generated contigs unambiguously assigned transposon locations}

To assess the quality of the assembly with respect to repetitive elements, we compared the number and position of Tc1 transposons in the him-9; ruIs32 MinION generated contigs to the 


\begin{tabular}{lrr} 
& \multicolumn{1}{l}{ Reads } & Percentage \\
\hline Total PF & $28,156,060$ & 100.00 \\
\hline Paired & $28,082,998$ & 99.74 \\
\hline Read 1 & $14,041,499$ & 49.87 \\
\hline Read 2 & $14,041,499$ & 49.87 \\
\hline Aligned & $26,581,603$ & 94.41 \\
\hline Properly Paired & $26,343,266$ & 99.10 \\
\hline Singletons & 69,881 & 0.26 \\
\hline Secondary Alignments & 73,062 & 0.27 \\
\hline Supplementary Alignments & 0 & 0.00 \\
\hline Duplicates & 584,096 & 2.20
\end{tabular}

Table 2: Illumina sequencing Read Data 

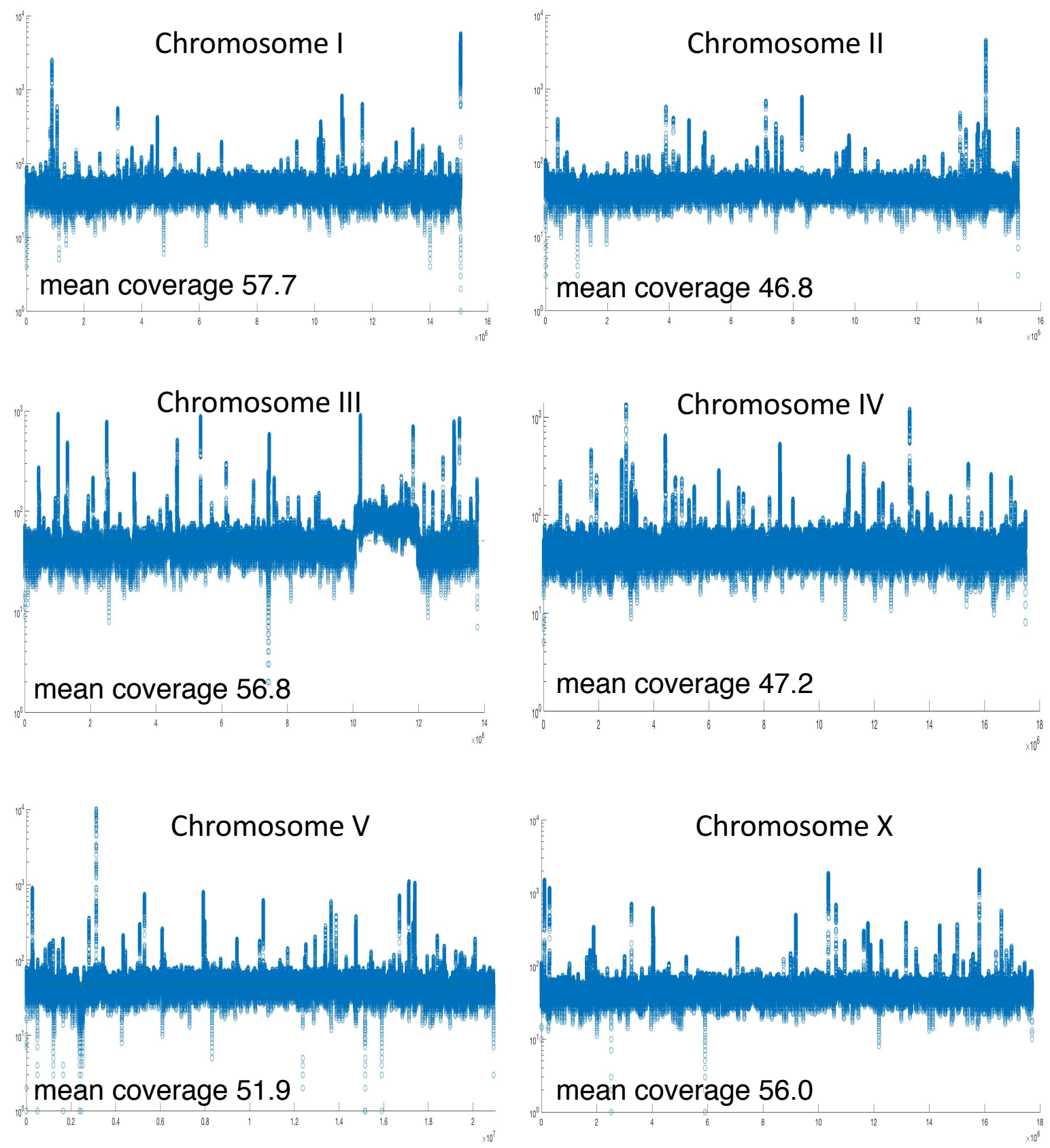

Figure 2: MinION read coverage (log scale) aligned to reference genome. Note the duplication between 10,062,096 and 11,973,739 on chromosome III. 
bioRxiv preprint doi: https://doi.org/10.1101/099143; this version posted January 30, 2017. The copyright holder for this preprint (which was not certified by peer review) is the author/funder, who has granted bioRxiv a license to display the preprint in perpetuity. It is made available under aCC-BY-NC-ND 4.0 International license.

\begin{tabular}{|c|c|c|c|c|c|}
\hline & Canu(HQ) & Canu(All) & Canu(HQ)+Pilon & Canu(All)+Pilon & Illumina $(>030)$ \\
\hline Total Bases & $2,184,632,490$ & $6,341,521,060$ & $2,184,632,490$ & $6,341,521,060$ & $4,022,919,599$ \\
\hline Nanopore data $>1 \mathrm{~kb}$ & $2,117,363,533$ & $6,025,477,392$ & $2,117,363,533$ & $6,025,477,392$ & \\
\hline Illumina data & & & $8,036,947,435$ & $8,036,947,435$ & $3,545,850,569$ \\
\hline Unassembled Data & $433,367,624$ & $1,086,588,269$ & & & $49,124,114$ \\
\hline \# contigs & 308 & 294 & 308 & 294 & 38,645 \\
\hline Total Assembly (bp) & $101,997,097$ & $103,164,837$ & $104,756,867$ & $106,194,296$ & $117 \mathrm{Mb}$ \\
\hline $\mathrm{N} 50(\mathrm{Mb})$ & 1.30 & 1.17 & 1.33 & 1.21 & 0.026 \\
\hline Average Contig Size (Kb) & 331 & 351 & 340 & 361 & 3 \\
\hline Average Read Length & 6,160 & 5,528 & & & $\sim 127(141 \mathrm{p} 1,112 \mathrm{p} 2)$ \\
\hline Read N50 & 8,692 & 7,672 & & & $\sim 169(181 \mathrm{p} 1,157 \mathrm{p} 2)$ \\
\hline Largest Contig (Mb) & $5,018,199$ & $4,573,586$ & $5,130,018$ & $4,688,558$ & $1,801,795$ \\
\hline Genome Fraction (\%) & 92.37 & 92.84 & 98.13 & 98.92 & 94.03 \\
\hline Total Aligned Length & $91,240,752$ & $91,290,952$ & $99,568,344$ & $100,288,126$ & $94,504,201$ \\
\hline \#Mismatches & 34,610 & 23,631 & 51,131 & 50,146 & 29,289 \\
\hline \#Indels & $1,551,278$ & $1,761,037$ & 64,364 & 61,304 & 6,831 \\
\hline Indels length & $2,413,421$ & $2,699,872$ & 185,846 & 181,052 & 34,903 \\
\hline GC\% & 36.36 & 36.44 & 36.00 & 36.07 & 37.43 \\
\hline Identity to reference & $97.31 \%$ & $97.01 \%$ & $99.76 \%$ & $99.77 \%$ & $99.93 \%$ \\
\hline
\end{tabular}

$\mathrm{HQ}$

2D pass from Chip84,85,89,90 and 1D pass from Chip94,95

All

All reads (1D template, $1 D$ complement $\& 2 D$ from pass and fail)

Table 3. C.elegans him-9 mutant Genome Assemblies and Polishing. Different genome assemblies using Nanopore, Illumina and combined data sets. (HQ) 2D pass from Chip84, 85, 89, 90 and 1D pass from Chip94, 85. (All) All reads, 1D template, 1D complement \& 2D from pass and fail catagories. 
Chromosome I

Chromosome II

Chromosome III

Chromosome IV

Chromosome V

\begin{tabular}{|c|c|c|c|c|c|c|c|c|}
\hline CONTIG & LENGTH & $\begin{array}{l}\text { PILON } \\
\text { LENGTH }\end{array}$ & CONTIG & LENGTH & $\begin{array}{l}\text { PILON } \\
\text { LENGTH }\end{array}$ & CONTIG & LENGTH & $\begin{array}{l}\text { PILON } \\
\text { LENGTH }\end{array}$ \\
\hline 0128 & 641,577 & 667,188 & 0093 & $1,404,993$ & $1,452,753$ & 1829 & 322,164 & 335,057 \\
\hline 0162 & 249,830 & 261,633 & 0066 & $1,825,969$ & $1,884,875$ & 1827 & 145,979 & 151,731 \\
\hline 0146 & 101,839 & 105,941 & 0190 & 145,554 & 149,288 & 0157 & 449,989 & 464,710 \\
\hline 0155 & 90,893 & 94,925 & 0113 & 522,805 & 538,113 & 0044 & 286,814 & 299,041 \\
\hline 0060 & 910,770 & 955,944 & 1732 & $1,147,314$ & $1,180,765$ & 1753 & 106,886 & 110,089 \\
\hline 0144 & 285,340 & 305741 & 1776 & $1,333,788$ & $1,365,313$ & 0063 & $1,054,603$ & $1,105,484$ \\
\hline 0041 & 147,174 & 159,766 & 1777 & 15,658 & 15,848 & 0121 & 780,480 & 817,746 \\
\hline 1812 & 597,398 & 614,699 & 1778 & $2,891,883$ & $2,957,296$ & 0031 & $1,971,186$ & $2,029,091$ \\
\hline 1811 & 19,219 & 19,306 & 1789 & 15,231 & 15,270 & 1735 & 55,591 & 55,699 \\
\hline 1725 & $4,573,586$ & $4,688,558$ & 0023 & 595,982 & 609,952 & 1736 & $2,020,242$ & $2,069,914$ \\
\hline 1729 & 318,637 & 327,171 & 1766 & $2,478,930$ & $2,559,627$ & 1798 & 159,446 & 162,596 \\
\hline 1727 & 83,750 & 85,600 & 0009 & $1,108,459$ & 1,153,611 & 1783 & $2,577,988$ & $2,640,414$ \\
\hline 0018 & 750,497 & 770,051 & 0119 & 327,432 & 341,061 & 0040 & 447,466 & 460,463 \\
\hline 0136 & 681,659 & 708,475 & 1819 & 18,180 & 18,661 & 0065 & $1,618,827$ & $1,687,451$ \\
\hline 0152 & 422,339 & 438,644 & 1806 & 371,509 & 383,023 & 1790 & 771,310 & 804,548 \\
\hline 0097 & $1,353,294$ & $1,397,343$ & 1805 & 24,717 & 25,461 & 1836 & 17,002 & 17,100 \\
\hline 0156 & 394,056 & 410,474 & 1804 & 550,140 & 573,165 & 1788 & 692,834 & 717,085 \\
\hline 0068 & 110,325 & 116,857 & & & & 1758 & 3,917 & 3,923 \\
\hline 1813 & $1,030,425$ & $1,069,619$ & & & & 0808 & 7,998 & 8,057 \\
\hline 1814 & 75,058 & 75,451 & & & & & & \\
\hline
\end{tabular}

\begin{tabular}{|r|r|r|r|r|r|}
\hline & 1771 & $1,395,592$ & $1,441,393$ \\
\hline
\end{tabular}

(2)

\begin{tabular}{|c|c|c|c|c|c|c|c|c|}
\hline $\begin{array}{c}\text { Total } \\
\text { CONTIG }\end{array}$ & 12837666 & 13273386 & $14,778,544$ & $15,224,082$ & $13,490,722$ & $13,940,199$ & $19,771,273$ & $20,406,552$ \\
\hline $\begin{array}{c}\text { Reference } \\
\text { bp }\end{array}$ & $15,072,434$ & & $15,279,421$ & $13,783,801$ & $17,493,829$ \\
\hline
\end{tabular}

Table 4. Worm Contigs 
bioRxiv preprint doi: https://doi.org/10.1101/099143; this version posted January 30, 2017. The copyright holder for this preprint (which was not certified by peer review) is the author/funder, who has granted bioRxiv a license to display the preprint in perpetuity. It is made available under aCC-BY-NC-ND 4.0 International license.

\begin{tabular}{|c|c|c|c|}
\hline & \multicolumn{3}{|c|}{ Contigs after Pilon } \\
\hline $\begin{array}{c}\text { Chromosome } \\
\text { (Size in bp) }\end{array}$ & $\begin{array}{c}\text { Pairwise } \\
\text { Identity (\%) }\end{array}$ & $\begin{array}{c}\text { Identical } \\
\text { Sites (\%) }\end{array}$ & $\begin{array}{c}\text { Reference } \\
\text { Coverage (\%) }\end{array}$ \\
\hline I (15,072,434) & 80.9 & 95.0 & 99.8 \\
\hline II $(15,279,421)$ & 83.3 & 96.1 & 99.9 \\
\hline III $(13,783,801)$ & 84.5 & 94.4 & 99.2 \\
\hline IV $(17,493,829)$ & 85.3 & 96.3 & 99.8 \\
\hline V $(20,924,180)$ & 85.7 & 96.0 & 99.2 \\
\hline X $(17,718,942)$ & 84.1 & 97.5 & 99.5 \\
\hline Mito (13,794) & 98.5 & 98.8 & 100 \\
\hline
\end{tabular}

Table 5. LASTZ alignment of contigs to the Six chromosomes and the C.elegans mitochondrial genome. 

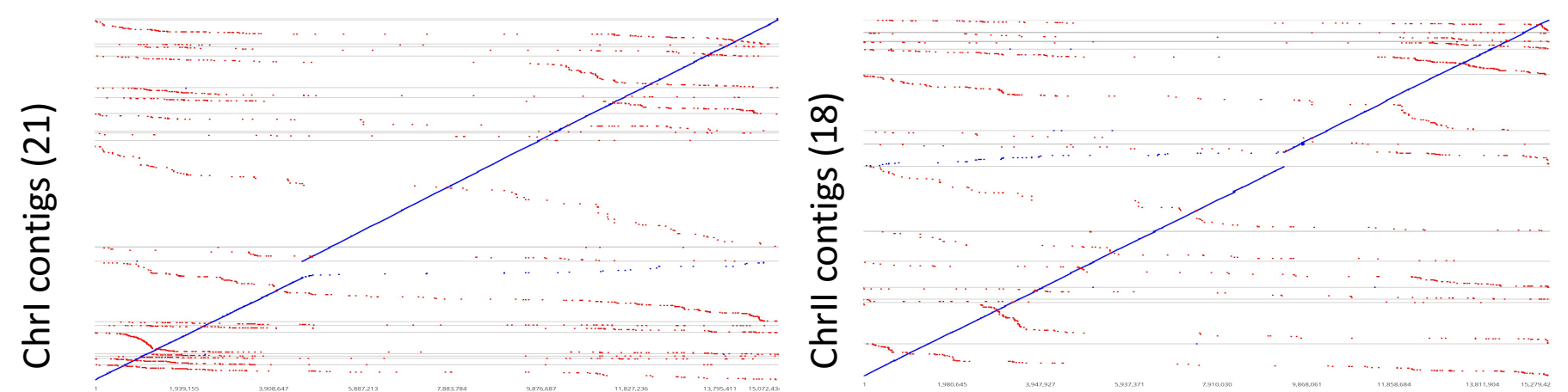

Chromosome I
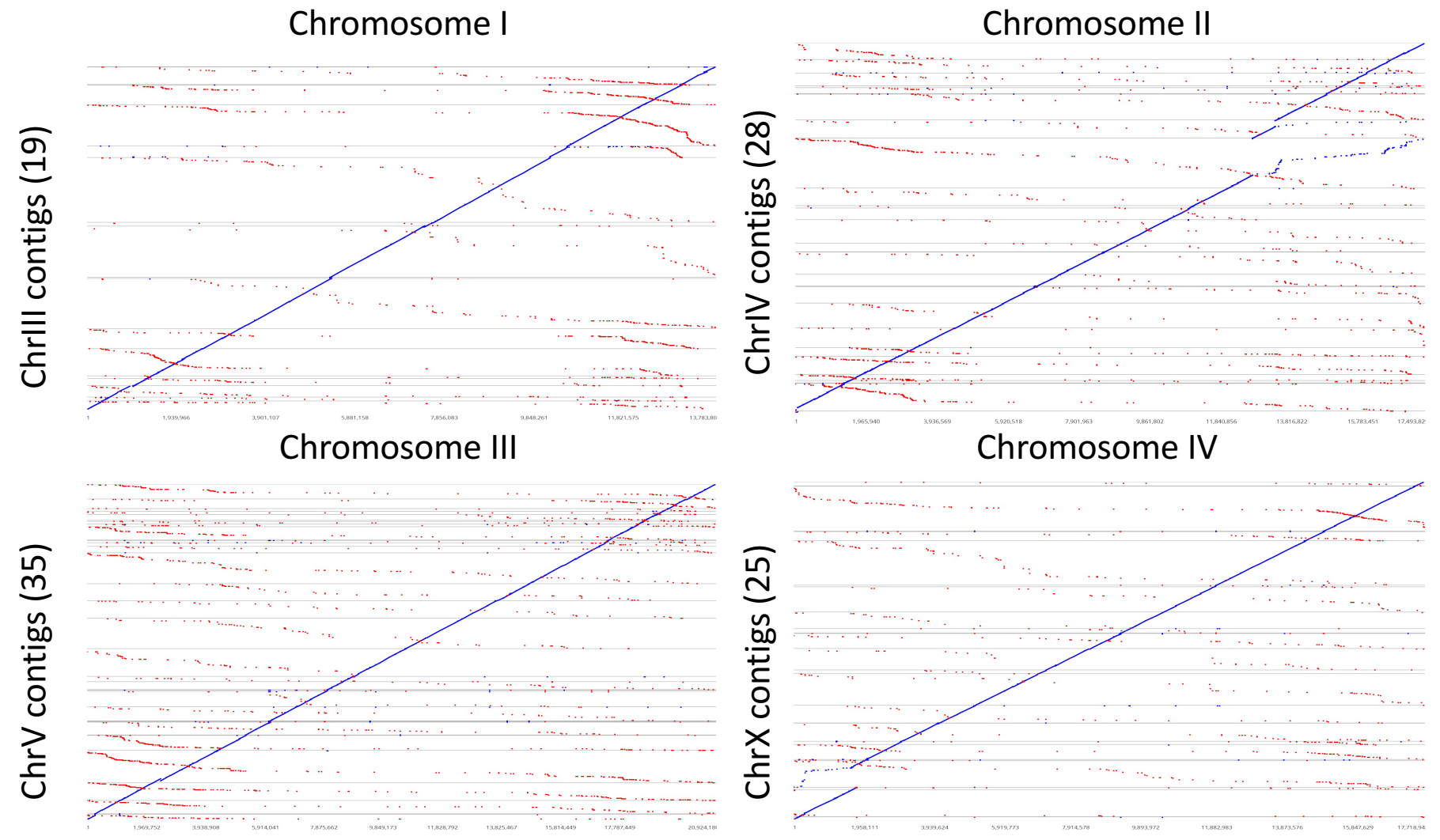

Chromosome V

Chromosome $\mathrm{X}$

Figure 3: LASTZ alignments of MinION contigs to $C$. elegans reference chromosomes. 
reference genome. Tc1 is a $1.6 \mathrm{~kb}$ transposon. NGS paired end reads are not sufficiently long to span the Tc1 transposons and cannot be unambiguously mapped resulting in a mapping quality score of 0 when aligned by the BWA aligner. Transposon number and position in the assembled contigs can be used as a measure of how effective MinION long reads are for spanning dispersed repeat regions. BLAST was used to align Tc1 reference sequences to the 145 worm contigs and the C. elegans reference genome. All described Tc1 elements were present in the MinION contigs and corresponded to their position in the reference genome based on the LASTZ alignments. (Table 6)

\section{MinION reads elucidated the structure of complex repetitive chromosome rearrangements}

In addition to de novo genome assembly, long sequencing reads can also facilitate the delineation of complex chromosomal rearrangements that contain multiple breakpoints, duplications, deletions and repeated sequences. The $C$. elegans strain sequenced here possessed two different complex genome rearrangements: him-9(e1487) is an acetaldehyde-induced duplication-insertion event on chromosome II and ruIs32 is a biolistic-mediated transgenic insertion on chromosome III. Previous data from oligo array hybridization, reverse transcriptase PCR, and inverse-PCR experiments suggested that him-9(e1487) was an insertion of approximately $20 \mathrm{~kb}$ of sequence from the $m a b-3$ region into the $x p f-1$ gene. Assembly of MinION reads revealed a significantly more complex genomic rearrangement. While the insertion breakpoints were consistent with previous data, the insertion was larger than previously anticipated. In addition to the mab-3 duplicated region, the insertion also included an inverted repeat of part of the mab-3 duplication and the second exon of $x p f-1$, resulting in a much larger, more complex insertion than expected (Figure 4A). We were able to use paired end reads and copy number variations in the Illumina data to confirm the multiple breakpoints and copy number variations of the mab-3 region (Figure 4B).

The biolistic-mediated insertion was located on contig tig00000045, which aligned to the right arm of chromosome III consistent with the published location for ruIs32 (Wormbase). From the MinION read assembly, it appears that the insertion contains three copies of the Ppie$1:: G F P:: H 2 B::$ pie-1 transgene and two copies of the ampicillin gene from the plasmid and two 
bioRxiv preprint doi: https://doi.org/10.1101/099143; this version posted January 30,2017 . The copyright holder for this preprint (which was not certified by peer review) is the author/funder, who has granted bioRxiv a license to display the preprint in perpetuity. It is made available under aCC-BY-NC-ND 4.0 International license.

\begin{tabular}{|c|c|c|c|c|c|c|c|c|}
\hline \multicolumn{5}{|c|}{ MinION Contigs } & \multicolumn{4}{|c|}{ Reference Genome } \\
\hline Contig Name & Chr & Length & $\begin{array}{c}\% \text { Pairwise } \\
\text { Identity }\end{array}$ & $\begin{array}{c}\% \text { Query } \\
\text { Coverage }\end{array}$ & Chr & Length & $\begin{array}{l}\text { \% Pairwise } \\
\text { Identity }\end{array}$ & $\begin{array}{c}\% \text { Query } \\
\text { Coverage }\end{array}$ \\
\hline tig00000076 & I & 1,610 & 99.6 & 100 & I & 1,611 & 99.6 & 100 \\
\hline tig00000097 & I & 852 & 99.3 & 53 & I & 852 & 99.3 & 53 \\
\hline tig00000097 & I & 741 & 99.7 & 46 & I & 741 & 99.7 & 46 \\
\hline tig00000148 & 1 & 1,532 & 98.5 & 100 & $\mathrm{I}$ & 1,611 & 99.6 & 100 \\
\hline tig00000066 & II & 1,598 & 98.8 & 100 & II & 1,612 & 99.8 & 100 \\
\hline tig00000093 & II & 1,598 & 98.9 & 100 & II & 1,611 & 99.9 & 100 \\
\hline tig00000093 & II & 1,608 & 99.7 & 100 & II & 1,612 & 99.6 & 100 \\
\hline tig00001732 & II & 862 & 99.7 & 53 & II & 861 & 99.7 & 53 \\
\hline tig00001766 & II & 1,600 & 98.8 & 100 & II & 1,611 & 99.6 & 100 \\
\hline tig00001766 & II & 1,605 & 99.2 & 100 & II & 1,611 & 99.6 & 100 \\
\hline tig00001778 & II & 1,605 & 99.3 & 100 & II & 1,611 & 99.8 & 100 \\
\hline tig00001778 & II & 1,609 & 99.1 & 100 & II & 1,611 & 99.4 & 100 \\
\hline tig00000009 & II & 1,473 & 99.1 & 91 & II & 1,472 & 99.3 & 91 \\
\hline tig00000063 & III & 1,613 & 99.4 & 100 & III & 1,611 & 99.6 & 100 \\
\hline tig00001790 & III & 1,599 & 99.1 & 100 & III & 1,611 & 99.7 & 100 \\
\hline tig00000055 & IV & 1,610 & 99.6 & 100 & IV & 1,610 & 99.6 & 100 \\
\hline tig00000084 & IV & 1,582 & 98.0 & 100 & IV & 1,611 & 99.6 & 100 \\
\hline tig00000099 & IV & 1,608 & 99.4 & 100 & IV & 1,611 & 99.6 & 100 \\
\hline tig00001760 & IV & 1,602 & 99.2 & 100 & IV & 1,611 & 99.7 & 100 \\
\hline tig00001793 & V & 1,511 & 99.0 & 94 & V & 1,522 & 99.7 & 94 \\
\hline tig00001793 & V & 1,603 & 99.2 & 100 & V & 1,611 & 99.7 & 100 \\
\hline tig00001785 & V & 1,595 & 98.6 & 100 & V & 1,611 & 99.4 & 100 \\
\hline tig00000017 & V & 1,609 & 99.6 & 100 & V & 1,612 & 99.3 & 100 \\
\hline tig00001801 & V & 1,607 & 99.3 & 100 & V & 1,611 & 99.6 & 100 \\
\hline tig00001737 & V & 725 & 98.9 & 45 & V & 729 & 99.5 & 45 \\
\hline tig00001737 & V & 1,610 & 99.1 & 100 & V & 1,610 & 99.6 & 100 \\
\hline tig00000005 & V & 1,504 & 99.3 & 94 & V & 1,508 & 99.6 & 94 \\
\hline tig00000039 & V & 1,602 & 99.1 & 100 & V & 1,611 & 99.8 & 100 \\
\hline tig00000039 & v & 1,595 & 98.8 & 100 & V & 1,611 & 99.8 & 100 \\
\hline tig00000039 & v & 1,602 & 99.3 & 100 & V & 1,611 & 99.6 & 100 \\
\hline tig00001739 & $\mathrm{V}$ & 1,596 & 98.8 & 100 & V & 1,611 & 99.6 & 100 \\
\hline tig00000211 & $x$ & 1,602 & 99.1 & 100 & $x$ & 1,611 & 99.6 & 100 \\
\hline tig00000074 & $x$ & 1,613 & 99.3 & 100 & $x$ & 1,611 & 99.4 & 100 \\
\hline
\end{tabular}

Table 6. Tc1 transposon numbers, locations and match compared to reference Genome. 
bioRxiv preprint doi: https://doi.org/10.1101/099143; this version posted January 30, 2017. The copyright holder for this preprint (which was not certified by peer review) is the author/funder, who has granted bioRxiv a license to display the preprint in perpetuity. It is made available under aCC-BY-NC-ND 4.0 International license.

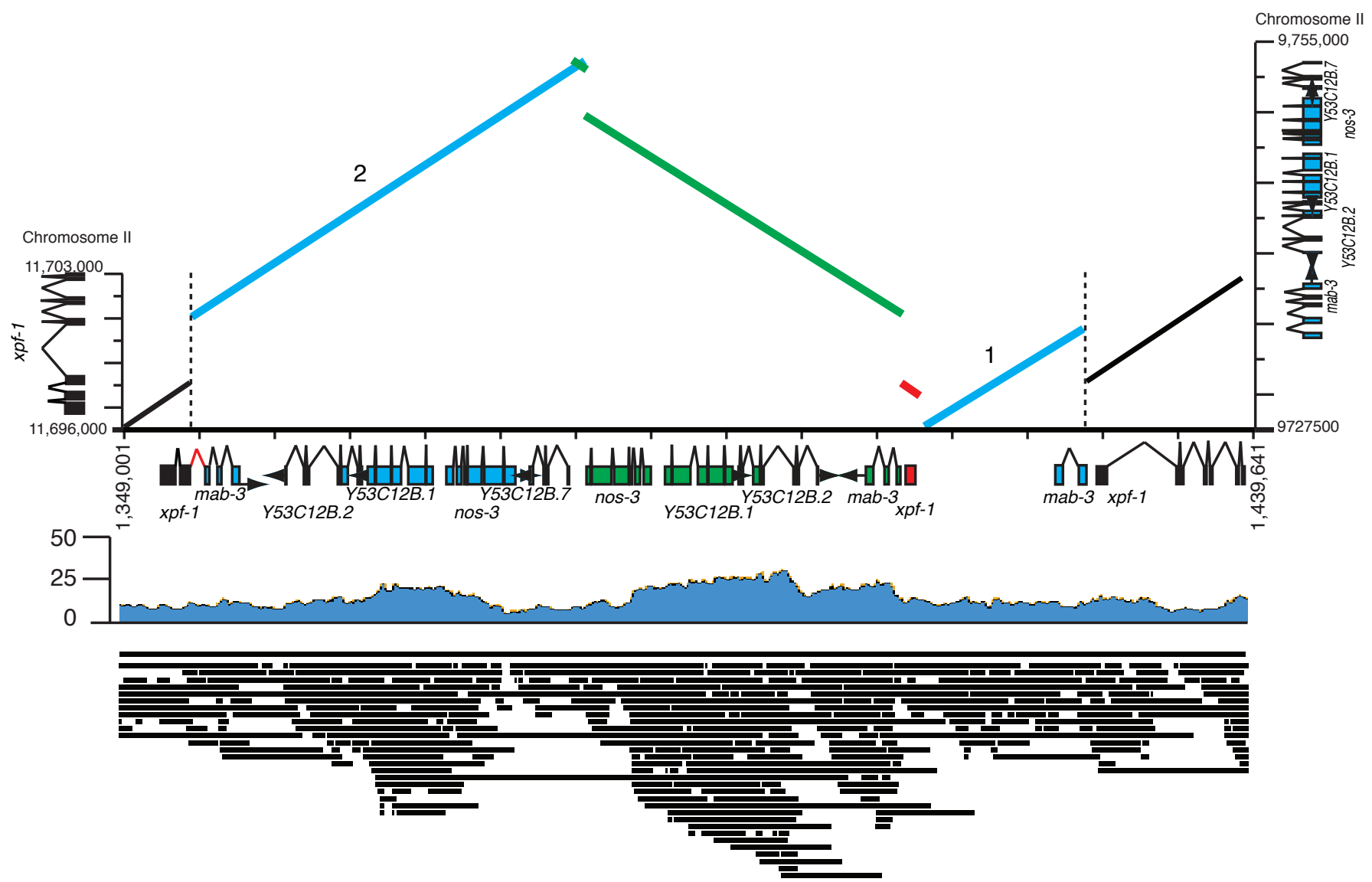

Figure 4A: Schematic of the 40kb complex insertion of mab-3 region into the xpf-1 gene. The insertion is in contig 1766. The insertion is an inverted repeat.Shown below are selected MinION reads mapping to the region spanning the various breakpoints.

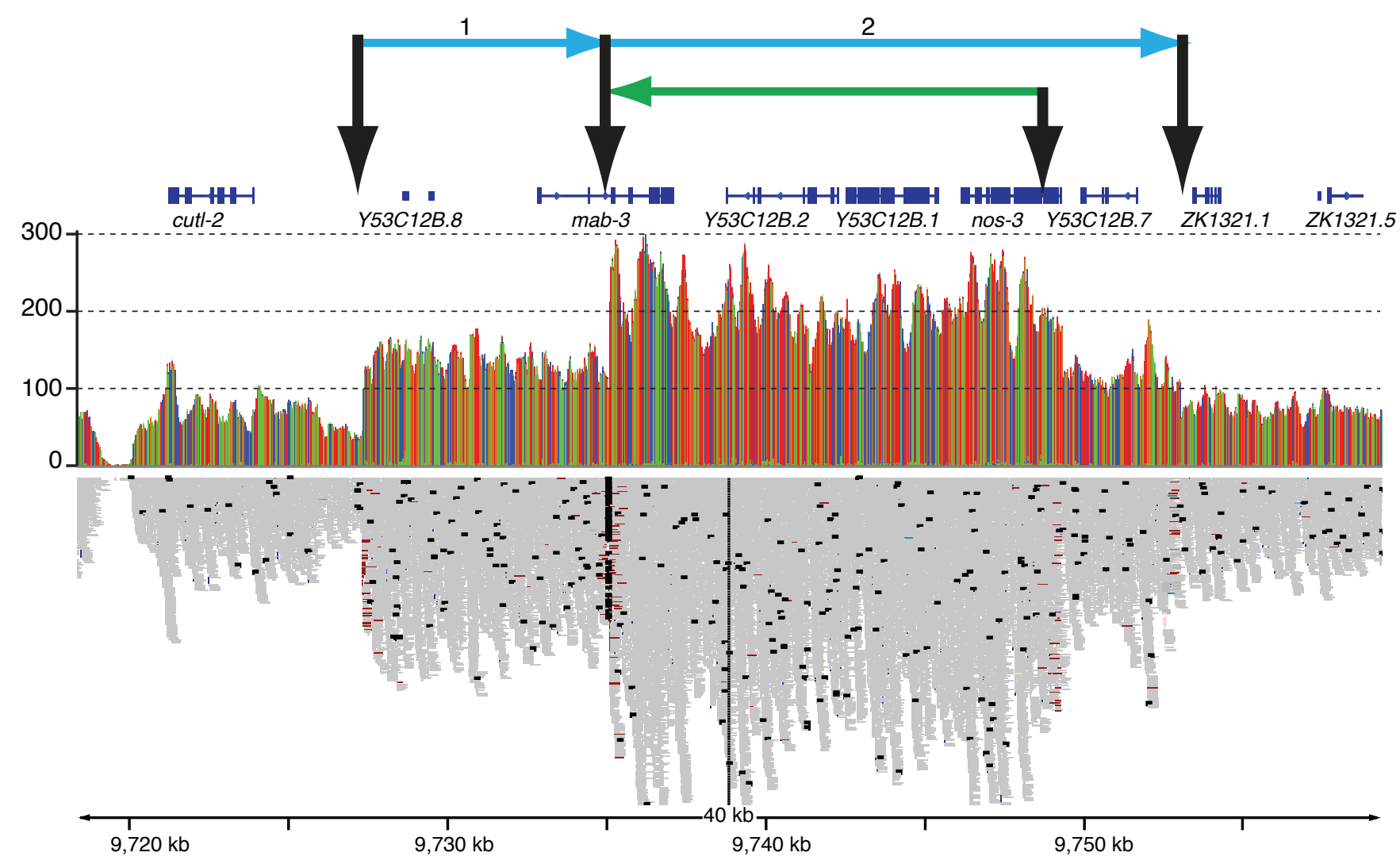

Figure 4B: The mab-3 region showing breakpoints detecting in MinION sequencing data and Illumina read coverage. 
partial copies of the unc-119(+) gene from the unc-119 transgene. The structure of the insertion is complex, with the $u n c-119(+)$ sequence interspersed within the plasmid sequence suggesting a complex integration event (Figure 5A). Copy number changes and breakpoints in pie-1 and unc119 were confirmed by the Illumina sequencing reads (Figure 5B). The integration event also appears to have generated a large duplication of approximately $2 \mathrm{Mb}$ of DNA (chrIII:10,062,096-11,973,739) from the region near the insertion site (Figure 2). Given the position of the insertion, the wild type unc-119 transgene should be genetically linked to the unc119(ed3) mutation and should not be lost through outcrossing. Indeed, we were able to ascertain both the mutant and two wild type transgenic alleles of unc-119 from the MinION contigs (Figure 5C).

\section{Discussion}

Advances in long read sequencing have opened new avenues for genome analysis. The relatively short sequencing reads produced by NGS allow for resequencing less complex, non-repetitive regions in well-characterized genomes. However, long sequence reads span repetitive regions and facilitate contiguous assemblies of large contigs. Similarly, long reads are essential for resolving complex chromosomal alterations such as those observed in tumour genomes. The major challenges previously limiting the use of long read TGS technology have been throughput, accuracy, and cost. The MinION sequencer has low equipment and consumable costs, and here we show that the MinION has achieved throughput and error rates that make it a viable option for the sequencing of novel genomes and genomes containing complex chromosomal rearrangements such as those observed in tumours. Continued advancements in both nanopore chemistry and basecalling will further improve throughput and accuracy. Additionally, with the advent of the high throughput ONT PromethION instrument genomes of larger sizes should be within easy reach.

We demonstrate here that the sequencing and de novo assembly of a large, low percent-GC (35.44), complex metazoan genome can be accomplished using MinION technology. We were able to assemble a near complete $C$. elegans genome with large contigs from less than 60 -fold 
bioRxiv preprint doi: https://doi.org/10.1101/099143; this version posted January 30, 2017. The copyright holder for this preprint (which was not A certified by peer review) is the author/funder, who has granted bioRxiv a license to display the preprint in perpetuity. It is made available under aCC-BY-NC-ND 4.0 International license.

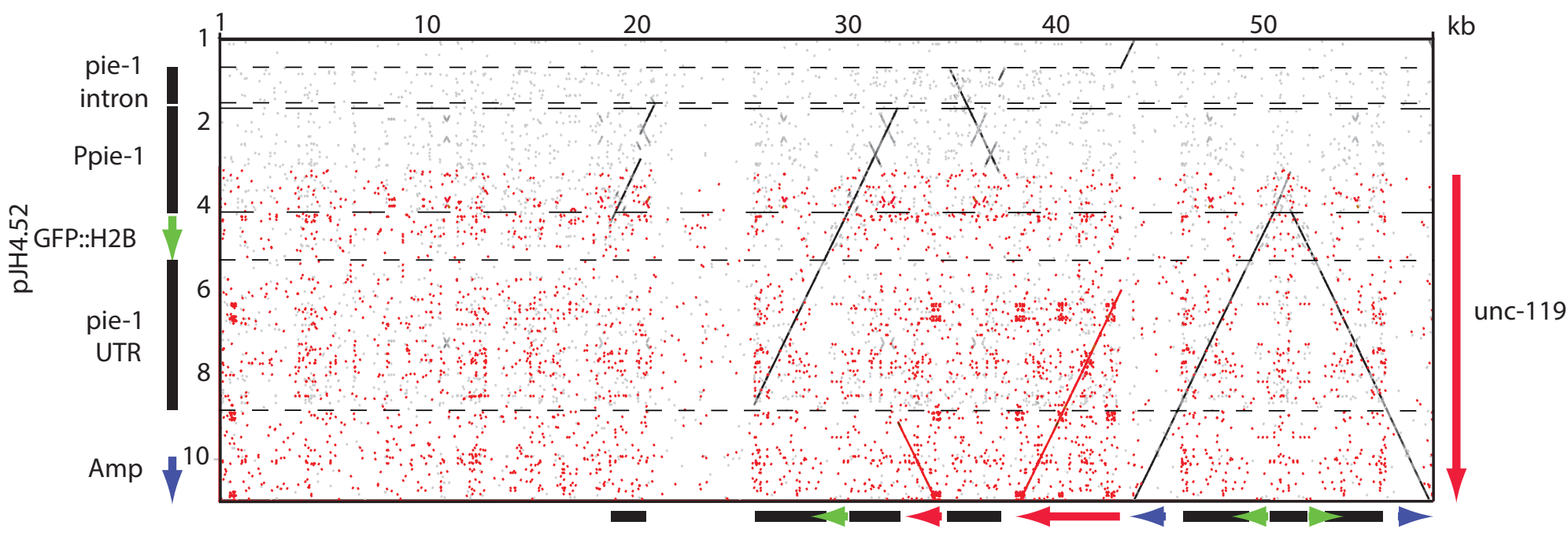

B

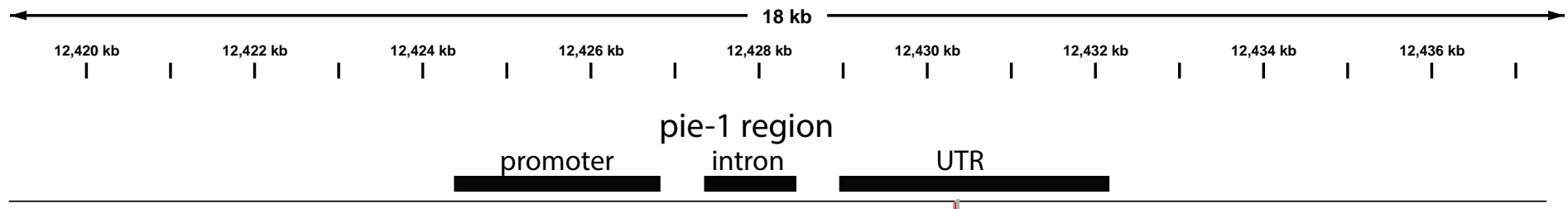
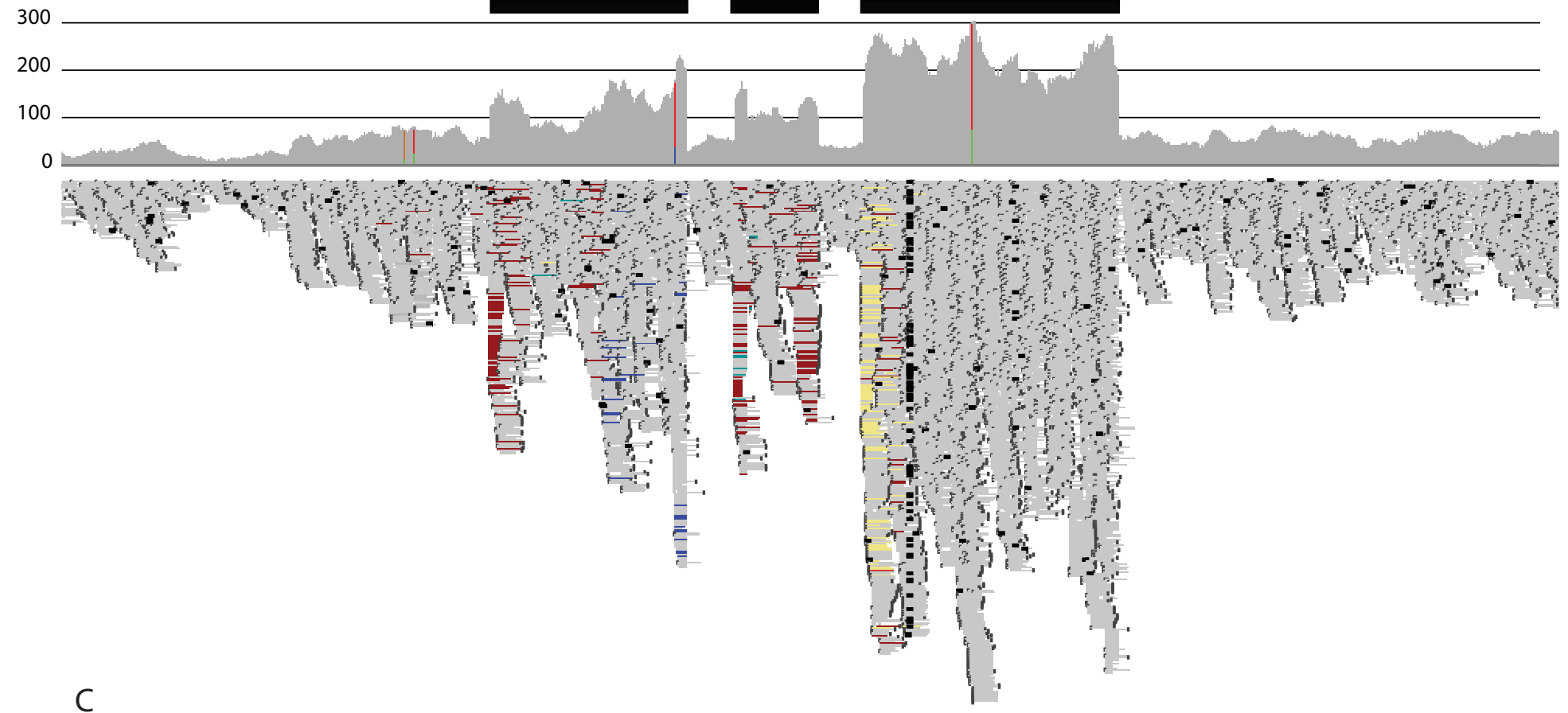

unc-119(ed3) GAATCGGCAAGATATGTCCGATATTGATTTGCGCCGAATTTTCT tig00000045 ${ }^{420101}$ GAATCGGCAAGATATGTCCGATATCGATTTGCGCCGAATTTTCT ${ }^{410144}$ tig00000045 ${ }^{426904}$ GAATCGGCAAGATATGTCCGATATCGATTTGCGCCGAATTTTCT ${ }^{426861}$ tig00000053 $3^{314809}$ GAATCGGCAAGATATGTCCGATATTGATTTGCGCCGAATTTTCT ${ }^{314852}$

Figure 5. Elucidating the ruIs32 insertion. (A) Dot plot of the contig 45 region that aligns with the pJH4.52 plasmid (pJH4.52 was used because pAZ132 sequence is not available. pAZ132 was derived from pJH4.52) and the unc-119 gene. (B) Illumina read data illustrating the breakpoints and copy number changes identified in the MinION data. (C) The sequence of unc-119 identified in the MinION data. 
sequence coverage. MinION sequence read quality has increased to a level that $1 \mathrm{D}$ reads now achieve error rates $<10 \%$ and result in more unique reads per flow cell.

Despite having higher error rates than NGS data, nanopore reads could be assembled into contigs with high concordance to the reference sequence. While consensus sequencing corrects for random sequencing errors, not all sequencing errors generated by MinION are random. Presently, MinION reads do not discern homopolymeric nucleotide tracts longer than 5 nucleotides. Consistent with this deficiency, the MinION assembled contigs did not contain any of the $396>18$ mer G-tracts known to be present in the C. elegans genome (Zhao et al. 2007). Pilon can improve the identity of homopolymeric tracts but not completely. Although homopolymeric runs were truncated in the MinION reads, the quality of sequence in their flanking regions was not affected. Illumina sequencing was more effective in sequencing homopolymeric tracts although we observed that Illumina reads were also affected by large Gtracts with lower read coverage in regions spanning G-tracts. Improvements in the ability to detect homopolymeric sequences from nanopore data are anticipated near-term.

For the purposes of this analysis, we assembled the genome using Canu alone and did not use manual finishing methods to bridge contigs. Assembled contigs were very large with an N50 contig size $>1 \mathrm{Mb}$. A more contiguous genome assembly could be generated from the same data by using manual finishing approaches to bridge contigs. For example, it is clear from the LASTZ alignment that many of the contigs contain sequence overlaps between adjacent contigs. The genome assembly could be made more complete using long reads that extend from the ends of the contigs to find potential links between existing contigs resulting in a more contiguous genome assembly. The genome assembly could also be improved by changing the quality of the DNA sample before sequencing. Contig breakpoints were often in regions containing repetitive regions that were larger than the average read length. Increasing the number of reads longer than these repeat regions will facilitate the spanning of these regions. This could be accomplished by sequencing deeper or by preparing the genomic DNA to favour the formation of larger fragments. 
Chromosome rearrangements are common in tumours and the identification of rearrangements is important for tumour characterization and treatment. Detecting chromosome rearrangements such as duplications, deletions and translocations pose challenges for sequencing-based approaches. NGS can detect copy number changes and identify potential breakpoints. However, complex rearrangements with multiple breakpoints and duplications can prove difficult to reassemble from NGS reads. We demonstrate that long MinION reads provide context for assembly of duplicated rearranged sequence and can delineate complex chromosome rearrangements. MinION long reads offer the opportunity to cheaply identify genome rearrangements in tumours including the highly complex chromothripsis events which result in thousands of clustered localized chromosomal rearrangements (Leibowitz et al. 2015).

Our sequencing and assembly of the $C$. elegans genome demonstrates the advancing capabilities of the MinION sequencer. Throughput and accuracy of the MinION platform continues to improve and is approaching 5-10 Gb of sequence per flowcell, which would generate sufficient sequence to assemble the $100 \mathrm{Mb}$ C. elegans genome. The long read capabilities of MinION nanopore sequencing facilitate unambiguous assembly of chromosome structure, thereby eliminating the need for physical mapping. These properties allow for sequencing of new genomes or tumour genomes with structural chromosome changes. Combining MinION and Illumina sequencing currently can delineate the structure of novel genomes with higher baselevel certainty. Alternative techniques, for example, using nanopore only event based correction methods (Loman et al. 2015), offer improved accuracies independent of hybrid NGS sequence correction. Combining this with future improvements in basecaller performance is anticipated to further remove the need for SBS in regard to high sequence accuracy.

\section{Acknowledgement}

The authors would like to thank both Dr. Mark Akeson (MA) and Dr. Benedict Paten (BP) for supporting $\mathrm{MJ}$ and HEO, and for allowing use of the computer cluster to run assemblies and analyses. JRT and TPS thank the Canadian Institutes of Health Research (grant \#10677) and Brain Canada Multi-Investigator Research Initiative Grant with matching support from Genome 
British Columbia, the Michael Smith Foundation for Health Research and the Koerner Foundation for funding. NJO and PH thank the Canadian Cancer Society (grant 702975). MJ and HEO thank the National Human Genome Research Institute of the US National Institutes of Health for funding their PI's under award numbers HG006321 (MA), HG007827 (MA), and U54HG007990 (BP). We also thank Oxford Nanopore Technologies for access to hardware, software and sequencing chemistries. 


\section{References}

Bessereau JL. 2006. Transposons in C. elegans. WormBook: 1-13.

C. elegans Sequencing Consortium. 1998. Genome sequence of the nematode C. elegans: a platform for investigating biology. Science 282: 2012-2018.

Coulson A, Huynh C, Kozono Y, Shownkeen R. 1995. The physical map of the Caenorhabditis elegans genome. Methods Cell Biol 4896100433: 533-550.

Coulson A, Kozono Y, Lutterbach B, Shownkeen R, Sulston J, Waterston R. 1991. YACs and the C. elegans genome. Bioessays 13: 413-417.

Coulson A, Waterston R, Kiff J, Sulston J, Kohara Y. 1988. Genome linking with yeast artificial chromosomes. Nature 335: 184-186.

Deamer D, Akeson M, Branton D. 2016. Three decades of nanopore sequencing. Nature Biotechnology 34:518-524.

Goodwin S, Gurtowski J, Ethe-Sayers S, Deshpande P, Schatz MC, McCombie WR. 2015. Oxford Nanopore sequencing, hybrid error correction, and de novo assembly of a eukaryotic genome. Genome Res 25(11):1750-1756.

Hodgkin J, Horvitz HR, Brenner S. 1979. Nondisjunction Mutants of the Nematode CAENORHABDITIS ELEGANS. Genetics 91: 67-94.

Jain M, Fiddes IT, Miga KH, Olsen HE, Paten B, Akeson M. 2015. Improved data analysis for the MinION nanopore sequencer. Nat Methods 12: 351-356.

Jain M, Olsen HE, Paten B, Akeson M. 2016. The Oxford Nanopore MinION: delivery of nanopore sequencing to the genomics community. Genome Biology 17: 239-250.

Koren S, Walenz BP, Berlin K, Miller JR, Phillippy AM. 2016. Canu: scalable and accurate long-read assembly via adaptive k-mer weighting and repeat separation. bioRxiv.

Leibowitz ML, Zhang CZ, Pellman D. 2015. Chromothripsis: A New Mechanism for Rapid Karyotype Evolution. Annu Rev Genet. 49:183-211. 
Loman NJ, Quick J, Simpson JT. 2015. A complete bacterial genome assembled de novo using only nanopore sequencing data. Nat Methods 12(8):733-735.

Li H. 2013. Aligning sequence reads, clone sequences and assembly contigs with BWA-MEM. $\operatorname{arXiv}[q-b i o G N]$.

Meier B, Cooke SL, Weiss J, Bailly AP, Alexandrov LB, Marshall J, Raine K, Maddison M, Anderson E, Stratton MR, Gartner A, Campbell PJ. 2014. C. elegans whole-genome sequencing reveals mutational signatures related to carcinogens and DNA repair deficiency. Genome Res. 24:1624-1636.

Norris AL, Workman RE, Fan Y, Eshleman JR, Timp W. 2016. Nanopore sequencing detects structural variants in cancer. Cancer Biol Ther 17(3):246-253.

Nurk S, Bankevich A, Antipov D, Gurevich A, Korobeynikov A, Lapidus A, Prjibelsky A, Pyshkin A, Sirotkin A, Sirotkin Y, et al. 2013. Assembling Genomes and Mini-metagenomes from Highly Chimeric Reads. In Research in Computational Molecular Biology: 17th Annual International Conference, RECOMB 2013, Beijing, China, April 7-10, 2013. Proceedings. (ed. Deng M, et al.), pp. 158-170. Springer Berlin Heidelberg, Berlin, Heidelberg.

Thompson O, Edgley M, Strasbourger P, Flibotte S, Ewing B, Adair R, Au V, Chaudhry I, Fernando L, Hutter H, et al. 2013. The million mutation project: A new approach to genetics in Caenorhabditis elegans. Genome Res. 23(10):1749-62.

Wei S, Williams Z. 2016. Rapid short-read sequencing and aneuploidy detection using MinION nanopore technology. Genetics 202:37-44.

Walker BJ, Abeel T, Shea T, Priest M, Abouelliel A, Sakthikumar S, Cuomo CA, Zeng Q, Wortman J, Young SK, et al. 2014. Pilon: an integrated tool for comprehensive microbial variant detection and genome assembly improvement. PLoS One 9: e112963.

Youds JL, O'Neil NJ, Rose AM. 2006. Homologous recombination is required for genome stability in the absence of DOG-1 in Caenorhabditis elegans. Genetics 2006 Jun;173(2):697-708.

Zhao Y, O'Neil NJ, Rose AM. 2007. Poly-G/poly-C tracts in the genomes of Caenorhabditis. BMC Genomics 8: 403. 


\section{Figure Legends}

Figure 1: Dot plots of LASTZ alignments on MinION Pilon polished contigs to the C. elegans reference chromosomes. * denotes contig that aligns to chromosome II and V.

Figure 2: MinION read coverage (log scale) aligned to reference genome. Note the duplication between 10,062,096 and 11,973,739 on chromosome III.

Figure 3: LASTZ alignments of MinION contigs to $\mathrm{C}$. elegans reference chromosomes. Discontinuities are due to contigs mapping to two different chromosomes. We have included these hybrid contigs on both chromosomes to allow for alignment. These hybrid contigs are most likely the result of contig assembly errors in highly repetitive regions.

Figure 4: Elucidating the structure of the complex him-9(e1487) acetaldehyde-induced mutation. (A) Schematic of the $40 \mathrm{~kb}$ insertion of the $m a b-3$ region into the $x p f-1$ gene based on contig 1701. The insertion structure suggests a mutation event in which the insertion was duplicated to generate an inverted repeat of the mab-3 region and the insertion point with $x p f-1$ (in red). (B) The mab-3 region with breakpoints denoted with arrows. Breakpoints detected by MinION are corroborated by Illumina read coverage and paired-end read alignment.

Figure 5: Elucidating the ruIs32 insertion. (A) Dot plot of the contig 45 region that aligns with the pJH4.52 plasmid (pJH4.52 was used because pAZ132 sequence is not available. pAZ132 was derived from pJH4.52) and the unc-119 gene. (B) Illumina read data illustrating the breakpoints and copy number changes identified in the MinION data. (C) The sequence of unc-119 identified in the MinION data. 